\title{
International perspective on mixed health care: Japan
}

\author{
Hisayuki Hamada, Samuel Lapalme-Remis
}

Japan's health care system should be the envy of the world. Japan consistently places near the top of the World Health Organization's overall health rankings, and does so while spending the smallest proportion of its GDP on health care than any member of the G7 (8\% in 2004; Canada spent 9.9\%), making it perhaps the world's best health care bargain. This accomplishment can be traced back to the founding of Japan's national universal insurance health care program in 1961, in which all Japanese could receive equal treatment at any health care facility. This system, however, is in danger of crumbling.

In Japan, patients must pay approximately $30 \%$ of their medical expenses (including medications) out of their pocket. In 2004, however, this still left the proportion of all health care costs borne by governments at an exceptionally high 81.5\% (Canada: 69.8\%). Particularly expensive are the country's drug costs, which make up $18.9 \%$ of all medical expenses (Canada: 17.7\%). The universal insurance system does exclude certain medical services such as orthodontia and cosmetic surgery; these uninsured services must be paid for out of pocket and are effectively privatized. Japanese law is very strict about the distinction between the provision of insured and uninsured care and prohibits medical institutions from providing both insured and uninsured services as different components of a single series of medical treatments.

Since the late 1990's, however, there has been increasing pressure from the business sector for the government to allow a mixed system in which providers could offer different medical services at the same time, some covered under the public-insurance system and

*To whom correspondence should be addressed: Hisayuki Hamada

Department of Medical Education

National Hospital Organization Nagasaki Medical Center

Kubara 2-1001-1

Omura City, Nagasaki Prefecture

Japan 852-8562

hhamada@nmc.hosp.go.jp some not. Foreign countries such as the United States have also pressured Japan to introduce market mechanisms and competition to medical care, allowing new insurance plans and medical-service businesses to flourish. In addition, wealthy individuals have expressed a wish to have access to high-level care at the very cutting edge of technology.

In 2001, Prime Minister Jun'ichiro Koizumi formed the Council on Fiscal and Economic Policy to bring Japan's public finances to order, aggressively promoting government-budget reform based on neoliberal principles. With the government's new emphasis on small government, free-market ideology, market-based incentives and increased privatization, Japan's social welfare programs faced major changes. Health care was no exception.

In 2004, a law was passed allowing private companies to participate in running health care institutions under limited parameters in exceptional cases only. Such involvement was restricted to six areas that were already under the category of uninsured medical services and represented cutting-edge medicine:

1) PET scans and other diagnostic imaging

2) Regenerative medicine

3) Medical genetics

4) Cosmetic surgery

5) In-vitro fertilization

6) Others

In 2005, a cosmetic-surgery venture company in Kanagawa Prefecture signed a collaborative contract with a university and became the first such private company to be founded.

However, in response to strong opposition by the Japan Medical Association and concerns by the general population, the Ministry of Health, Labour and Welfare took a cautious approach to the growing number of private companies entering the health care sector throughout Japan and issued a number of reservations 
regarding increased privatization of health care. First, there is a danger that because the medical services required by patients may not be those most profitable to private companies, important medical services may not be made sufficiently available. Second, medical services might no longer be provided in certain geographical regions if service providers withdraw from less profitable areas. Finally, the cost of medical services might increase considerably. In effect, despite the law, private companies are still prevented from forming national chains of health care facilities and the removal of the ban on mixed health care never occurred.

This lack of movement has done nothing to change the fact that the Japanese health care system faces increasingly difficult challenges year by year. By 2005, the proportion of the Japanese population older than 65 years old had exceeded $20 \%$ and the birth rate sat at its lowest level ever, 1.26. As a result, Japanese society is fast becoming the oldest society in human history, and the long-predicted collapse of the public healthinsurance system is becoming reality. Between 1985 and 2002, the total annual cost of health care in Japan nearly doubled from 16 to 31.1 trillion yen. This funding is split as follows: $51.7 \%$ comes from the universal health insurance contributions (shared between employers and employees), $15.3 \%$ comes directly from the patient's pocket, $25.1 \%$ is paid for by the national government, and $7.9 \%$ is paid for by local governments. Yet with an cost increase of 1 trillion yen (CDN\$8.7 billion) per year (after the USA, the highest rate of increase in the world), it is difficult to see where the needed funding will come from.

Japan has one of the highest per-capita number of hospitals in the world, with 8.4 hospital beds per 1000 population (Canada: 3.0 ; USA: 2.8). It is not uncommon for a single physician to run his own hospital, adding to the excess. This is seen as one of the major causes of the unrelenting increase in health care costs. While the government is making an effort to decrease the number of beds that are located in small- and medium-sized hospitals, there is great resistance among the population to dramatic changes in bed distribution, making it necessary for the government to tread carefully. On the other hand, there is a shortage of physicians, with only 2.0 per 1000 population (Canada: 2.1). This problem has been exacerbated in rural areas due the residentmatching system introduced in 2004, which has accelerated the tendency of young doctors to concentrate in urban areas. Rural areas are increasingly likely to lack the necessary doctors and hospitals.

Given this state of crisis, calls for the development of private medicine within the areas permitted by law, as well as for increased application of uninsured and mixed medical care, are increasing among government, business, some physicians (approximately 50\% of physicians working in hospitals according to a recent survey) and a minority of patients (about 20\%). However, the July 2007 House of Councillors election dealt a crushing loss at the polls to the ruling Liberal Democratic Party that had promoted aggressive fiscal reform, including health-care reform. The majority remains opposed to the increased application of market ideology and free-market competition. The election result highlighted the fact that the population wishes to maintain the current universal health-insurance plan, which it perceives as fair and affordable.

On November 8, 2007, an individual patient successfully sued the government in the Tokyo District Court by claiming that the government violated his constitutional rights by refusing to allow him to apply his public health insurance to any of his cancer treatment because part of his treatment had included uninsured services. The government had insisted that such prohibited "mixed" treatment relieved it of its duty to pay even for the insurable portion of his treatment. In effect, the court ruled that the government's suppression of a mixed system was unconstitutional. In response to this ruling, the Japan Medical Association and patient groups expressed concern that the provision of medical care would now depend on patients' financial status. Other patient groups applauded the decision. The decision is sure to fan the flames of the debate regarding the removal of the blanket ban on mixed health care.

Based on recent political events, it is clear that the general population in Japan is in favour of maintaining the present national universal insurance health care system. As a result, the debate on the adoption of increased uninsured or mixed medical care is at a standstill. With the nation in a state of political gridlock, there is little indication that battling political parties will be able to come up with any effective solutions to controlling the rapidly escalating costs that threaten to destroy the health care system voters are so eager to preserve.

Hisayuki Hamada, MD, PhD, is Chief of Medical Education at the National Nagasaki Medical Center. He studied Canadian public health and family medicine at the University of Toronto from 2004 to 2006 as an official of the Japanese Ministry of Health, Labour and Welfare.

Samuel Lapalme-Remis, MA, is a medical student at McGill University and Executive Focus Editor of the McGill Journal of Medicine. Prior to his studies in medicine, he received a Master of Arts in Japanese Language and Society from the University of Sheffield. 